\title{
Article \\ Measurement of Intraoperative Blood Loss in Minimally Invasive Neurosurgery
}

\author{
Sindhuja Krishnamoorthy ${ }^{1}$, Branesh M. Pillai ${ }^{2}$, Bibhu Sharma ${ }^{2}$ and Sorayouth Chumnanvej ${ }^{1 * \text { (i) }}$ \\ 1 Neurosurgery Division, Department of Surgery, Faculty of Medicine Ramathibodi Hospital, Mahidol \\ University, Bangkok, Thailand. ; sindhuk995@gmail.com \\ 2 Center for Biomedical and Robotics Technology (BART LAB), Department of Biomedical Engineering, Faculty \\ of Engineering, Mahidol University, Salaya, Thailand. ; branesh.mad@mahidol.ac.th, \\ sharmabibhu2@gmail.com \\ * Correspondence: sorayouth.chu@mahidol.edu; Tel.: +662-441-4255 Fax: +662-441-4254
}

\begin{abstract}
Surgeries that take place in medicine and dentistry or during any form of childbirth results in a significant amount of blood loss. The prevalent measurement methods that surgeons and anesthesiologists utilize as the "gold - standard" have several drawbacks. There are numerous other methods to measure blood loss, which, however, due to their impracticality and limitations, are not ideal either. This paper focuses on minimally invasive neurosurgery in particular, by taking into account a surgical technique known as Endoscopic Endonasal Transphenoidal surgery (EETS), which is used to treat pituitary tumors and adenomas. Along with the review of the existing literature pertaining to the blood loss management, this paper proposes a modified electrode probe method along with the concept of usage, computer interface, and the system of integration. The probe is intended to measure the hematocrit count from the collected blood under all circumstances, such that the medical practitioner is assisted to improve the blood loss management technique for better patient recovery.
\end{abstract}

Keywords: Endoscopic Endonasal Transphenoid (EET); Blood Loss Measurement, Electrochemical Measurement

\section{Introduction}

Blood loss during any type of surgery is one of the biggest problems faced in the OR today. Out of the estimated 313 million surgical procedures that are done worldwide annually, recent estimates show that at least 4.2 million people die within the 30 days of surgery per annum, which accounts for $7.7 \%$ of all deaths [1]. Perioperative bleeding remains a major risk during and after surgery and is associated with a high rate of death, complications, and healthcare resource use. "Advances in anesthesia, surgery, and transfusion medicine over the past decade have led to the development of 'patient blood management', a multimodal, evidence-based strategy consisting of three pillars: treating anaemia, reducing perioperative blood loss [2], and improving tolerance to anaemia".

Blood loss management varies for different circumstances, such as those in dentistry, gynaecology, anesthesiology, and surgeries for all other causes. Irrespective of that, anaemia being the most common outcome in surgical practices necessitates blood transfusion, which has further risk factors [3]. There are several management methods such as tourniquets, antifibrinolytics, cell salvage, drains, surgical technique, and topical agents that are used currently to reduce blood loss. In terms of measurement, various methods are also utilized, although sparingly, due to inaccuracies, expensiveness, inefficiency, and higher time consumption. Hence, visual estimation remains the most convenient and prevalent blood loss measurement method in surgical settings. Both surgeons and anesthesiologists utilize this method in blood loss management regardless of the risk factors and inaccuracies.

These issues are unequivocally relevant to the case of neurosurgery due to congruency in blood loss measurement, management, and collection among most of the surgeries. 
Surgeons and anesthesiologists utilize the same varieties of methods, among which, visual estimation is undoubtedly the most frequently used. In this paper, the case of Endonasal Endoscopic Transsphenoidal surgery (EETS) is particularly taken as a reference, in discussing blood loss management for experimental execution, data collection, and analysis.

\subsection{EET Background and Procedure}

The pituitary gland is a small pea-sized endocrine gland that is located at the bottom of the hypothalamus at the base of the brain. To be more precise, it is located above the nasal passage, lies within the thin bone structure called sella above the sphenoid sinus. It is known as the body's 'Master' gland as the main function involves secreting essential hormones that regulate homeostasis as well as manage other endocrine glands. Hence, when a tumor starts to develop in this gland, it is exceptionally detrimental. A brain tumor is a mass that is resulted from the abnormal growth of the brain cells which can be divided into two types: primary brain tumor and metastatic brain tumor. The primary brain tumor is distinguished as the growth within the brain itself and can either be benign or malignant.

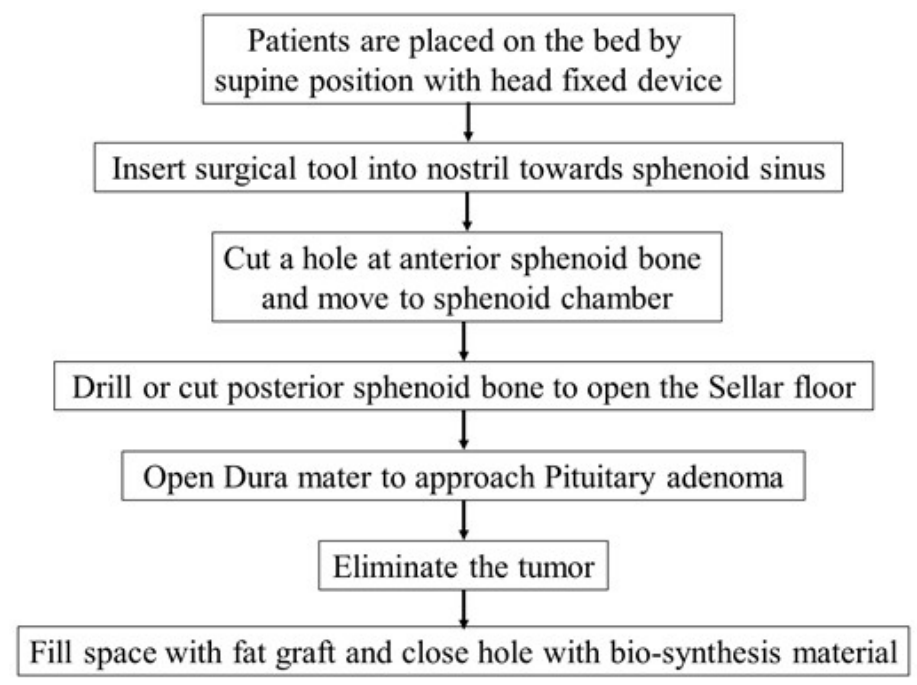

Figure 1. Flowchart of EET Procedure

The metastatic brain tumors are the cancer cells that spread to the brain via the bloodstream from another location in the body. Unlike primary tumors, all metastatic tumors are malignant and are more common in adults, whereas primary tumors occur more frequently in children. In regards to the pituitary gland, most pituitary tumors or pituitary adenomas are benign and they frequently develop in the front two - thirds of the gland which is a part of the anterior pituitary. These tumors are usually identified according to their size or functional status. In terms of size, adenomas that are less than 10 $\mathrm{mm}$ in size are known as micro adenomas and those that are larger than $10 \mathrm{~mm}$ are called macro adenomas. In terms of functionality, the tumor that produces excess hormones is deemed hyper functioning and those that produce no effect are deemed non-functioning adenomas.

The treatments for pituitary tumors and adenomas are dependent on several factors such as size, location, whether it is functioning or not functioning, patient age and the overall patient health. There are three main treatments for pituitary tumors: drug therapy, radiation and surgery. Radiation therapy is often used in addition to surgery and drug therapy, in which the goal is said to be to reduce and control the size of the tumor. There are numerous ways to surgically treat pituitary adenomas, among which Endonasal Endoscopic Transsphenoidal surgery is the most common method that is used as treatment without disturbing other regions within the brain or along the pathway leading to the gland. 
Endonasal Endoscopic Transsphenoial surgery is performed via the nose and the sphenoid sinus to remove tumors and cysts such as pituitary adenoma, Craniopharyngioma, Meningioma, Rathke's cleft cyst and Chordoma [4]. These surgeries are done in teams with neurosurgeons and ear, nose and throat surgeons with an endoscope and a sinus surgical tool that are inserted together through the nasal cavity, thus passing through and enlarging the sinus cavities. Usually there are various instrumentations used in the endoscopic technique such as the endoscope, the fiber optic cable, the light source, the camera, the monitor and the video recording system [5]. EET surgery procedures are only performed by expert surgeons with the help of an operative and perioperative checklist [6] as it is a relatively new technique and involves two complicated processes. Beginning with the sphenoid sinus approaching process, it is then followed by the tumor elimination process. The first step itself poses intricacy due to the fact that it lacks any anatomical landmark inside the nasal cavity, hence making it difficult to locate and it takes an average of 1 hour to complete. In terms of the second part, the complexity lies in the precise and accurate movement of surgical tools as it is used to operate inside the brain surrounded by brain tissue, thus making this process last an estimated time of 2-4 hours [7]. The surgical technique is composed of three stages, with the nasal being the first stage where the scope is inserted through the chosen nostril all the way to the sphenoethmoid recess and the sphenoidotomy is executed. Then during the sphenoid phase, several procedures are taken place such as the detachment of the nasal septum from the sphenoid rostrum, the removal of the sphenoid septum, anterior sphenoidotomy and the identification of the landmarks inside the sphenoid sinus [8].

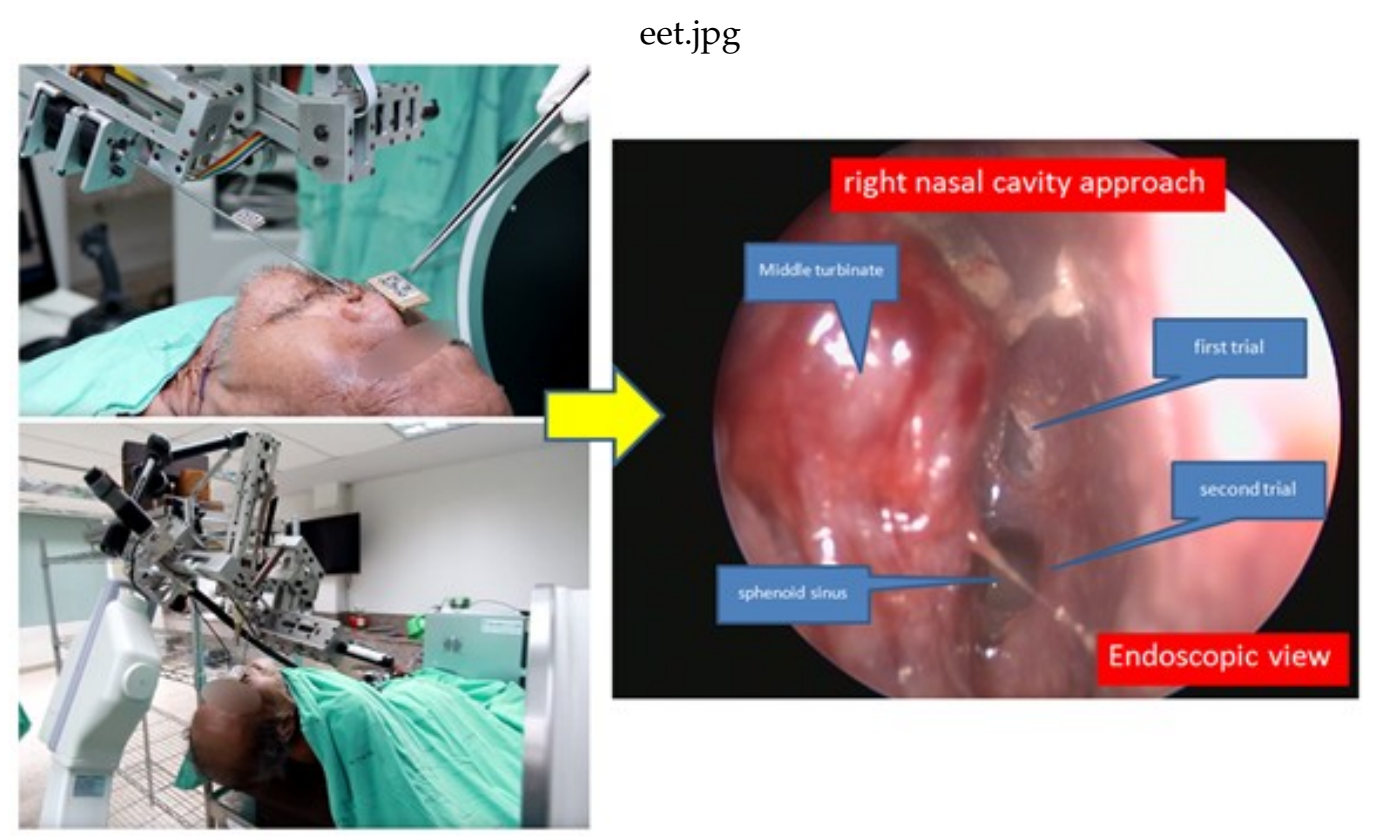

Figure 2. Cadaveric Experimentation of Robotic EET Surgery in Ramathibodi Hospital, Bangkok

Finally, in the sellar phase the sellar floor is opened to remove the tumor. After the lesion is removed, the entire procedure ends in covering the sella with bone graft or any other synthetic graft and removal of the endoscope from the nostril [9].As every surgical procedure is not devoid of inherent risks, meticulous process as that of EETS undoubtedly pose several of them. Primarily, the entire process requires high accuracy and any small deviations can increase the chances of damaging the surrounding anatomical landmarks. Also, the process is endangered by the probability of error in maintaining a stable trajectory throughout the surgery resulting in fatigue due to the incredulously long procedure [7]. 


\section{Literature Review}

\subsection{Types of Blood Loss measurements}

Currently in the Medical field, be it gynecology or orthopedics, there is no gold standard method in accurately quantifying blood loss. There are generally two ways in identifying blood loss, the direct method and the indirect method. The direct method involves methods used in collecting and quantifying blood, but not limited to, using swabs, suction device and post- operative drainage etc. The blood loss measurement in swabs largely consists of visual estimation, suction device involves the volume and weight from the suction device bottles and post - operative drainage includes drainage of blood from the operation site [10]. However, all these methods are not claimed as the standard technique due to their poor precision, reliability and validity and an inconsideration of haematomas or haemolysis, which account for most of the blood loss. The indirect method however is slightly more accurate and do take into account haematomas and haemolysis. These methods include, but are not limited to hemoglobin or hematocrit drop and transfusion volume, although they are somewhat related to one another. However, there lie the disadvantage, where in the two methods are but a reflection of blood loss [11].

Haemorrhaging continues to be the leading cause of maternal mortality and morbidity [9]. In a 2007 study, Sara S. et al stated that one of the major reasons behind such abundant deaths was related to the accuracy of quantifying blood loss. Quantification not only restores the volume of lost blood but is also vital for immediate management; it is necessary to measure the incidence of haemorrhage (PPH) and to monitor trends. It has also been noted that postpartum haemorrhage had been underestimated by $50 \%$ due to clinical difficulty in accurately calculating blood loss [12]. Accurately measuring blood loss is a huge challenge and it has been reported that there have been several underestimations in 5 out of 12 clinical scenarios. Besides, Sara S. et al also mentioned that in research conducted in Australia, midwives and other health professionals had underestimated blood loss by $30-50 \%$.

If there are such discrepancies in quantifying blood loss, immediate management and incident haemorrhaging can become even more complex than it already is [12]. In a study conducted in 2018, Diaz V et al verified that the most common method of estimating blood loss was visual estimation, as it always had been. However, for more accurate estimation, doctors do perform indirect blood collection. The lost blood is soaked by pads and other material, is contained in a disposable, funnelled, plastic collector bag, which is attached to a plastic sheet. As a gravimetric measurement method, all of the blood is weighed. Whereas, a direct method is when a 'calibrated delivery drape' is placed under the mother and is tied around her waist with the calibration delivery funnel portion hanging below between her legs. Although they provide more accurate results, they tend to be difficult to perform or are not readily accessible in most settings. Hence, the predisposition towards visual estimation seems plausible [13].

Similarly, in wisdom tooth surgery, postoperative bleeding is a major issue when it comes to achieving complete homeostasis due to the increased vascular nature of the tissues and the potential exposure of the open socket to the patient's exploring tongue and fingers. Bleeding for 12-24 hours post extraction is normal in healthy patients as clotting will commence. Managing bleeding involves using a damp sheet of gauze and placing it over the socket firmly for minutes until homeostasis is reached. If bleeding continues, the socket is rinsed with saline and the gauze is then replaced. The excess blood with saline is then suctioned into the canister. In another dental case presented by Akinbami et $\mathrm{al}$, on the oral and maxillofacial surgical procedure, it is mentioned that excessive blood loss is resulted from the facial microvasculature and major blood vessels [14]. Along with a significant amount of bleeding that can occur during dissection of the capillary-rich skin, subcutaneous tissue, and muscles in the maxillofacial region. At this time the most common method of maintaining homeostasis in such surgeries is the use of hypotensive anaesthesia and tranexamic acid [15]. A broad range of hemostatic agents is available as secondary measures to enhance homeostasis especially during long-lasting bleeding [16]. 
Other ways of managing blood loss in dentistry involve compressing the area with gauzes and/or cauterizing or ligating the vessels that are responsible for blood loss. Other drugs aside from Tranexamic acid include desmopressin, adrenaline, and more [17].

Surgeons and anaesthesiologists often disagree on the amount of blood loss postoperatively especially after long operations or those with a significant amount of bleeding. As most of the contemporary method of estimating blood loss is limited to visual estimation, other methods which are relatively accurate are time consuming and inefficient. Since most of the blood is collected in sponges, the most important function of the visual method is to estimate the amount of blood in sponges, gauzes, and towels regardless of the amount of blood that is soaked [18]. Most of the time surgeons dilute the blood in irrigation solutions and determine the blood loss accordingly. This causes the measurement of blood loss to be more subjective while the blood collected in the canister would be more accurate [19].

In Laparoscopic procedures, for example, in urologic surgery, blood loss is obtained via the suction system that contains a heparinized saline solution which is then collected in suction canisters. Surgeons carefully suction all surgical blood loss and any residual losses are suctioned through surgical drains into the canisters post-surgery. The final value is then calculated by subtracting the irrigation fluid volume from the total volume contained in surgical canisters. Usual methods of direct measurement are not entirely reliable because of the limitations and difficulties of accurately measuring whole blood loss, especially in surgeries that involve hidden losses [20] or difficult to measure losses [21]. In orthopedic surgery, the same shortcoming prevails where surgeons and anaesthesiologists use the eyeballing method. Other methods such as gravimetric, calculation, radiometric and direct spectrophotometry are utilized in measuring intraoperative blood loss too.

With the direct spectrophotometric method being considered a benchmark as this is used to measure intraoperative samples extracted from surgical sponges and suction canisters postoperatively. However, no matter how accurate this method may be, as compared to visual estimation, there is still up to a $10 \%$ error rate. For surgeries that result in major blood loss, allogeneic blood transfusion (ABT) is commonly performed for hemodynamic management, hence making accurate determination of blood loss a necessity. There are several complications that come with ABT and doctors often tend to keep this procedure to a minimum, however in order to do so an increase in the methods for blood management $[22,23]$. The following methods are the readily available approaches that are commonly used by doctors today:

\subsubsection{Visual Estimation}

As mentioned earlier it is one of the direct methods used in calculating blood loss and is very common despite the fact that it is highly inaccurate. It is utilized by several surgeons and anaesthesiologists from assessing surgical sponges, suction canisters, and the operating room environment until they come to a decision [22].

\subsubsection{Gravimetric}

As mentioned by Norris E. Lenahan et al., the gravimetric method was first introduced into the literature field by Wangensteen in 1942 [24], as he concluded that this method was a rapid and accurate process for measuring blood loss. The procedure involves weighing all the surgical sponges and estimating the lost blood by subtracting the weight before vs after use with every gram of weight equivocal to $1 \mathrm{~mL}$ of lost blood. This method though inexpensive, simple, and practical, it is neither precise nor accurate [25].

\subsubsection{Dilution}

A variation of these methods is the dilution method. In this method, first reported by Mollison and Veall, $851 \mathrm{Cr}$-labeled blood is transfused into the patient, and after allowing the blood to be evenly distributed over several minutes, a venous sample is obtained from the patient and the patient's circulating blood volume is calculated. By repetition of the procedure postoperatively, an accurate blood loss estimate can be made. Once again, 
however, the drawbacks of expense, complexity, and special equipment avert it from being a preferred method [26].

\subsubsection{Radiometric}

The radiometric method as first reported by Rankin et al labels the RBCs with radioactive chromium which is thus used by a gamma-radiation reading, both preoperative and postoperative. After the operation, any material used to collect blood is measured for gamma - radiation, resulting in an estimated blood loss. This method is quite accurate however quite complex, not economically efficient, and is the least preferred by patients [26].

\subsubsection{Volumetric}

Another method mentioned by Johar and Smith [26] is the volumetric method which consists of measuring preoperative and postoperative irrigation, with the difference representing blood loss. As stated by James McIvor "The volume of any fluid to be introduced into the operative field is measured pre-operatively and any excess fluid in the aspirator jar after the operation is assumed to be blood. Sources of error are aspirated fluids other than blood" [10].

\subsubsection{Colorimetric}

Primarily reported by Gatch and Little in 1924 and then refined by Hallberg and Nilsson in 1964, this method is quite accurate, inexpensive and reproducible and has been used by several researchers on blood loss during menstruation due to its efficiency [26]. In this technique the total blood collected in swabs, drapes, gauze, and any other items, and suction and drainage are washed in water and the change in color of the water is then used to measure blood loss. "This increase in redness is best measured using a photometer which operates at a frequency sensitive only to changes in blood concentration." The photometer measures the optical density of the mixture which is then converted into a blood loss reading.

\subsubsection{Photometry}

Photometric examination and lab testing have been demonstrated to be dependable systems for estimating blood loss and are currently talked about as the " gold standard" for its accuracy [10]. As per Paul C. Johnson et al., various forms of photometric methods include measuring the average red cell velocity whilst counting the number of red cells that pass per unit time, in other words, red cell flux. Hence when red cell flux is divided by its velocity, it results in the hematocrit index, which represents the average number of red cells per unit length of the flow column passing the photosensors. Another method involves measuring the average light absorption by the blood in the capillary, in other words, capillary opacity, which is then compared to the change when a red cell passes the sensor and is expressed as a fraction [27].

\subsubsection{Analytical Blood Loss Estimation}

This method involved the use of a meticulous measurement technique carried out within the best conditions for the measurement of blood loss. All blood loss formulae have similar structures and bases [21,28-30]. A euvolemic state between the pre and postoperative times is assumed. Based on this principle, blood loss is assumed to be correlated with the perioperative difference of hematocrit (or hemoglobin levels) and the patient's blood volume [21]. The essential difference among the formulae is how they correct for blood hemodilution using different types of functions: linear, proportional, or logarithmic functions. Hence, blood loss estimation formulae require a value of the patient's blood volume (estimated by specific formulae) and perioperative values of either hematocrit or hemoglobin levels. A selection of the main blood loss formulae is performed. 
The selection considered the most cited and the most recent formulae in the medical literature [21,28-30].

\subsubsection{Electrochemical}

The technique is a promising tool that is used to measure blood hematocrit (HCT) levels via electrical measurement, where the conductivity of blood is closely correlated with HCT. To be more specific, the impedance measurement and electrochemical impedance spectroscopy (EIS) of blood provide a more accurate method for determining hematocrit. The electrical properties of plasma and blood cells shed light on the basic details of the health status of patients. EIS allows the investigation of a number of other blood parameters in detail. This method is very promising for monitoring the glucose concentration, hemoglobin content, thrombus formation, erythrocyte aggregation and blood coagulation [31].

\subsubsection{Optical}

Optical techniques have been developed to provide the physiological blood information (e.g., oxygen saturation, hematocrit) and pathological blood information (e.g., thrombus, hemolysis) conveniently, continuously, and without making direct contact with the blood. Optical techniques have a lot of advantages in measuring levels of hematocrit. For example, we can measure the levels of hematocrit during the course of the treatment continuously. This continuous information of hematocrit allows us to predict symptoms including bleeding and thrombosis [32]. The significant advantage of optical measurements is the ability to measure a blood constituent continuously and in real-time to "literally see immediate results of therapy on a patient." Optical measurements have the ability to provide instantaneous feedback during surgery [33].

\subsubsection{Spectrometry}

"A more precise measurement of blood loss is haemoglobin concentration $(\mathrm{Hb})$ in venous blood sampling and spectrophotometry. With the dye dilution technique, a known quantity of dye is injected into the vein and its plasmatic concentration is monitored after the uterus stops bleeding. Using nuclear medicine, a radioactive tracer is injected, and its concentration is monitored after the uterus stops bleeding" [33]. In the study conducted by Philip Nowicki et al., "the spectrophotometric method using absorbance spectrophotometry can be considered a reasonable benchmark for assessment of blood loss but can practically only be done postoperatively [3,12]. Furthermore, although the spectrophotometric method is accepted as a standard reference method, there is a reported error range of up to $10 \% . "$ [22].

\subsection{Types of blood loss measurements in neurosurgery}

Neurosurgery, like head and neck surgery and orthopaedics, consists of major complications in terms of blood loss measurements as it requires large volumes of fluid to irrigate wounds during surgery [34]. In Craniosynostosis repair surgery, where there is considerable blood loss as reported by P.Meyers et al., large blood volumes are trapped in surgical drapes and dilution with irrigating fluids preventing precise measurement of blood loss. Estimation of blood loss during these procedures refers to weighing sponges and measurement of suction volume [34]. In a research conducted by Ayhan Ulusoy et al. regarding the adolescent idiopathic scoliosis surgery, a common spinal deformity, it is mentioned that, although there are several methods for estimating the perioperative blood loss, each of them is stricken with exigent limitations [19]. Anesthesiologists' commonly used method is a visual estimation, even though it was rendered most inaccurate, where they visually examine blood collected in suction canisters, surgical sponges, drapes, towels, and on other surfaces [35]. The types of blood loss measurement utilized in neurosurgery are not different from the types used in other surgeries such as those mentioned above. 


\section{Methodological Proposition}

In regards to the predicaments discussed through literature and practice, a method is proposed in this paper which involves haematocrit calculation using the electrochemical method. This is done with an electrode-probe which requires dipping into a test tube, or in this case a blood collection chamber, or even by simply placing one drop of blood onto the electrode surface. The method is fundamentally based on the concept initially proposed by Steuer et al. [32], which within 10 seconds, would offer high accuracy, reproducibility and still maintain the simplicity of usage and maintenance. The latter is for cases that involve blood volume that is not collected from suction, but from cloth and other free surfaces. The idea is based on the utilization of the electrical conductivity of the entire blood, excluding the blood that is mixed with saline and other types of fluids that may be mixed with the blood. Apart from that, certain provisions have also been amalgamated to this model including a method that ensures uniformity between observers [36], an alarm to send alerts due to the low power or poor solution-electrode contact, and therefore obtaining the haemoglobin values from the measured hematocrit value.

Several ideas have been incorporated in regards to the electrode, one of which is the bioelectrical impedance technique presented by Kichul Cha et al., which is based on the principle of the change of the impedance of biological tissue in regards to the water content. When this technique is applied to blood, high correlations between the resistivity or conductivity of blood and haematocrit have been reported [37]. Another idea that would be further assessed and utilized for the consideration of the electrode probe would be the notion developed by Myounggon Kim et al., in which they address the electrical properties of blood as a function not only of HCT [38] but also of the electrical conditions in the plasma, which is a huge factor that is dealt within ORs daily. They had prepared twelve blood samples such as four kinds of plasma conditions (hypotonic, isotonic, two kinds of hypertonic conditions) at three different HCT levels, in order to cover any and all possible electrical variables of plasma. Alongside, attempting to reduce measurement errors, their proposed HCT estimation parameter sheds light on the characteristics of both the changes in volume of red blood cells (RBCs) and electrical conditions of plasma, simultaneously [39]. These methods will be further explored and modified as per the proposed electrode-probe model to make it adept to this research setting and thus compatible with blood under any circumstance.

\section{Discussion}

The purpose of conducting this study is to explore a novel, accurate and effective method of detecting and measuring arbitrary blood loss during neurosurgery, which then can be potentially utilized in other surgeries. As mentioned earlier, several methods are in use, however, they are either impractical, time-consuming, inefficient, inaccurate, or expensive. Visual estimation is the prime method being used between the surgeon and the anaesthesiologist in the OR and it is highly dependent on blood loss found in suction canisters, surgical sponges, surgical drapes/gowns, and the operating room environment. Therefore, it is an inaccurate method of measurement [40], in which blood loss is underestimated [41] during times of high blood loss and overestimated when the blood loss volume is less [22]. This makes the patient susceptible to consequences that come with high blood loss, such as hypovolemic shock and the need for blood transfusions that in turn comes with their own set of consequences such as infections [42], hemolytic transfusion reactions, and in times of overestimations of blood loss, there is a risk of volume overload. For the ultimate patient care, an accurate method for blood loss is required with less time consumption and economically justifiable [43].

The intent of this study and of the proposed model is to create a methodology that produces immediate response in real-time, with user-friendly, economically efficient and most importantly, accuracy in results. As mentioned, the device must be able to produce results under any and all circumstances that blood is collected in, whether it is hypotonic, isotonic or hypertonic or whether the blood is mixed in any other solutions upon collection. 
The possible drawbacks at this point is quite uncertain as experimental procedures have to be performed in order to identify any further improvements. However, potential drawbacks would start with the practicality of producing a device that overcomes any and all hindrances regarding blood loss estimation from the collected blood along with tackling the flaws presented by other studies. Other potential drawbacks include the ways that blood is collected aside from the suction canisters, such as gauzes, drapes, sponges. As they present the issue of blood drying up, thus making it difficult for reading. Even if they are then soaked in saline solution or DI water, there is still a chance for some blood to be stuck to the fabric. Normal surgical practice may have to be altered in order to come out with results of $100 \%$ accuracy. Accuracy of the results will always be one to look out for as numerous parameters will be taken into consideration especially in the case of real-time.

The proposed methodology is a synergetic combination of several other similar methods that utilize the idea behind the electrode-probe design, that was initially drawn from Steuer et al., which provides the instantaneous result. However, a study conducted in 1983 addressed its limitations in that the conductivity not only depends on the p-parameters but also the medium. Several other limitations accompany this design and require advanced technology and biophysical specialization and familiarity with the complex impedance measuring principles as well as electrode design problems [44]. Later on, in a 1994 study, a four-electrode impedance method accurately determined haematocrit in a large number of patient blood samples. However, their limitations were regarding the calculation of results in real-time and in large quantities, its portability, and its ability to mask the haemodialysis method.

Several other electrode-probe methods calculate HCT levels using blood glucose, such as the one present in a 2016 study by Saeda and Kaneda [45] using biosensors. However, the limitations associated with this method are a decrease in accuracy due to increased electric power usage, complexity in the structure of usage, uncertainties due to additional procedures needed to calculate HCT, and it is mainly catered to whole blood. Thus the proposed model would be unique in the sense that it will produce accurate results not just in whole blood, but the blood that is mixed with various other fluids, as it normally is during intraoperative conditions.

\section{Conclusions}

The motivation behind the proposed model is the improvement of blood loss management by exploring and discovering new ways to accurately and efficiently measure arbitrary blood loss using the haematocrit parameter. The concept that has been developed through an elaborate investigation of the prevalent literature, would initially be implemented and tested in neurosurgery and eventually also be adapted into other surgical procedures, with an intention of improving patient care and recovery. The future implication of the electrode-probe model would be to conduct experiments using blood under several circumstances and varying concentrations. The probe is to be modified according to the methodological proposition adhering with minimum measurement error through the consideration of the impedance of blood and/or blood glucose. This section is not mandatory, but can be added to the manuscript if the discussion is unusually long or complex.

Author Contributions: Conceptualization, S.K. and S.C.; methodology, B.M. and S.C.; investigation, S.K. and B.S.; writing-original draft preparation, S.K., B.S.; writing-review and editing, B.M. and S.C.; supervision, S.C.; project administration, S.C.; funding acquisition, S.C.. All authors have read and agreed to the published version of the manuscript.

Conflicts of Interest: The authors declare no conflict of interest.

\section{References}

1. Shah, A.; AJR, P.; Klein, A. Strategies to minimize intraoperative blood loss during major surgery. British Journal of Surgery 2020, 107, 26-38.

2. Kabilan, A.; M, D.; Jain, A. Estimation of blood loss post-dental extraction. Drug Invention Today 2018, 10. 
3. Clevenger, B.; V, M.S.; Klein, A. Patient blood management to reduce surgical risk. British Journal of Surgery 2015, $102,1325-1337$.

4. Cappabianca, P.; Cavallo, L.; Divitiis, E. Endoscopic endonasal transsphenoidal surgery. Neurosurgery 2004, 55, 933-941.

5. Solari, D.; Villa, A.; Angelis, D. Anatomy and surgery of the endoscopic endonasal approach to the skull base. Translational Medicine@UniSa 2012,2, 36.

6. Christian, E.; Harris, B.; Wrobel, B. Endoscopic endonasal transsphenoidal surgery: implementation of an operative and perioperative checklist. Neurosurgical focus 2014, 37, 1 .

7. Chalongwongse, S.; S, C.; Suthakorn, J. Analysis of Endonasal Endoscopic Transsphenoidal (EET) surgery pathway and workspace for path guiding robot design. Asian journal of surgery 2019, 42, 814-822.

8. Lasio, G.; Ferroli, P.; Felisati, G. Image-guided endoscopic transnasal removal of recurrent pituitary adenomas. Neurosurgery 2002-07, 51, 136-7.

9. Cappabianca, P.; LM, C.; Divitiis, E. Endoscopic endonasal transsphenoidal surgery. Neurosurgery 2004, 55, 933-941.

10. McIvor, J. A method of assessing operative and post-operative blood loss. British Journal of Oral Surgery 1967, 5, 1-10.

11. Flordal, P. Measurement of blood loss in clinical studies. European Journal of Anaesthesiology (EJA 1997, $14,35-37$.

12. Buckland, S.; Homer, C. Estimating blood loss after birth: using simulated clinical examples. Women and Birth 2007, 20, 85-88.

13. Diaz, V.; E, A.; Carroli, G. Methods for blood loss estimation after vaginal birth. Cochrane Database of Systematic Reviews 2018, p. 9.

14. Akinbami, B.O.; Onajin-Obembe, B. Assessment of Intraoperative Blood Loss during Oral and Maxillofacial Surgical Procedures in a Nigerian Tertiary Health Care Center. Journal of Blood Transfusion 2014, 2014, 1-8. doi:10.1155/2014/301467.

15. Akinbami, B.; Onajin-Obembe, B. Assessment of intraoperative blood loss during oral and maxillofacial surgical procedures in a Nigerian tertiary health care center. Journal of blood transfusion 2014.

16. N, C.; F, F.; YDP, B. Bleeding in Dental Surgery. In Biosurgicals-The Next Frontier in Operative Approaches; IntechOpen, 2019.

17. Piñeiro, A.; Somoza, M.; Gandara, J. Blood loss in orthognathic surgery: a systematic review. Journal of Oral and Maxillofacial Surgery 2011, 69, 885-892.

18. Kabilan, A.; M, D.; Jain, A. Estimation of blood loss post-dental extraction. Drug Invention Today 2018, 10.

19. Ulusoy, A.; S, D.; Erdem, S. How accurate is visual estimation of perioperative blood loss in adolescent idiopathic scoliosis surgery? Acta orthopaedica et traumatologica turcica 2018, 52, 267-271.

20. H, J.; RA, H. Hidden blood loss in anterior lumbar interbody fusion (ALIF) surgery. Orthopaedics and Traumatology: Surgery and Research 2016, 102, 67-70.

21. Jaramillo, S.; Montane, M.; Capitan, D. Agreement of surgical blood loss estimation methods. Transfusion 2019, 59, 508-515.

22. Nowicki, P.; Ndika, A.; Kemppainen, J. Measurement of intraoperative blood loss in pediatric orthopaedic patients: Evaluation of a new method. Journal of the American Academy of Orthopaedic Surgeons 2018, 2.

23. Pennestrì, F.; Maffulli, N.; Sirtori, P. Blood management in fast-track orthopedic surgery: an evidence-based narrative review. Journal of orthopaedic surgery and research 2019, 14 .

24. Wangensteen, O. Controlled administration of fluid to surgical patients including description of gravimetric methods of determining status of hydration and blood loss during operation. Minnesota Med 1942, 25, 783-789.

25. Lenahan, N.; T, S.; Metcalf, D. Blood determination and estimation of blood loss during surgical operations. Archives of Surgery $1948,57,435-442$.

26. R, J.; R, S. Assessing gravimetric estimation of intraoperative blood loss. Journal of Gynecologic Surgery 1993, 9, $151-154$.

27. Johnson, P.; D, H.; Dial, J. Measurement of capillary hematocrit by photometric techniques. Microvascular research 1973, 5, 351-356.

28. Hurle, R.; Poma, R.; Maffezzini, M. A Simple Mathematical Approach to Calculate Blood Loss in Radical Prostatectomy. Urol Int 2004, 72, 135-139.

29. Lopez-Picado, P.; A, A.; Barrachina, B. Determination of Perioperative Blood Loss: Accuracy or Approximation? Anesthesia and Analgesia 2017, p. 125 -.

30. Gerdessen, L.; Meybohm, P.; S, C. Comparison of common perioperative blood loss estimation techniques: a systematic review and meta-analysis. J Clin Monit Comput 2020.

31. Zhbanov, A.; Yang, S. Electrochemical impedance spectroscopy of blood for sensitive detection of blood hematocrit, sedimentation and dielectric properties. Analytical Methods 2017, 9, 3302-3313.

32. Steuer, R.; DA, B.; Barrett, L. Optical measurement of hematocrit and other biological constituents in renal therapy. Advances in renal replacement therapy 1999, 6, 217-224.

33. Abbasi, S.; Khan, F.; Adil, S. Comparison of visual Estimation of Blood Loss with serial hemoglobin and hematocrit estimation in supratentorial craniotomy. Priory Lodge Education 2011, 14, 1-8.

34. Meyer, P.; Renier, D.; Arnaud, E. Blood loss during repair of craniosynostosis. BJA: British Journal of Anaesthesia 1993, 71, 854-857.

35. Mooney, J.; Barfield, W. Validity of estimates of intraoperative blood loss in pediatric spinal deformity surgery. Spine deformity 2013, 1, 21-24.

36. Yoong, W.; Karavolos, S.; Damodaram, M. Observer accuracy and reproducibility of visual estimation of blood loss in obstetrics: how accurate and consistent are health-care professionals? Archives of gynecology and obstetrics 2010, 281.

37. Cha, K.; Faris, R.; Brown, E. An electronic method for rapid measurement of haematocrit in blood samples. Physiological measurement 1994, 15.

38. Lee, H.; Barber, C.; Rogers, J. Electrochemical hematocrit determination in a direct current microfluidic device. Electrophoresis 2015, 36, 978-985. 
39. Kim, M.; Kim, A.; Kim, S. Improvement of electrical blood hematocrit measurements under various plasma conditions using a novel hematocrit estimation parameter. Biosensors and Bioelectronics 2012, 35, 416-420.

40. LD, R.; JM, L. Estimation of blood loss is inaccurate and unreliable. Surgery 2016, 160, 946-953.

41. GG, R.; P, S.; PV, V. Surgeons often underestimate the amount of blood loss in replacement surgeries. Chinese Journal of Traumatology 2014, 17, 225-228.

42. HA, P.; MP, B. Transfusion-associated infections: 50 years of relentless challenges and remarkable progress. Transfusion 2010, 50, 2080-2099.

43. Gross, J. Estimating allowable blood loss corrected for dilution. Anesthesiology: The Journal of the American Society of Anesthesiologists 1983, 58, 277-280.

44. Schwan, H. Electrical properties of blood and its constitutents: Alternating current spectroscopy. Blut: Zeitschrift für die Gesamte Blutforschung 1983, 46, 185-197.

45. Saeda, M.; Kaneda, H. Biosensor comprising electrode for measuring hematocrit value". US Patent 2016, 10309888. 